\title{
Evaluación de las alarmas de un sistema de monitoreo para una unidad de cuidados intensivos y especiales basándose en criterios de usabilidad
}

\author{
M.Pineda Arango ${ }^{\psi}$, E.Aguilar Ceballos, M.A.Suárez Echeverri, J. G. Barreneche \\ Bioinstrumentation and Clinical Engineering Research Group - GIBIC, Bioengineering Department, \\ Engineering Faculty, Universidad de Antioquia UdeA, Medellin, Colombia
}

\begin{abstract}
Resumen - En este artículo se presenta la aplicación de un modelo de pruebas de usabilidad a un sistema de monitoreo de signos vitales usados específicamente en Unidades de Cuidados Intensivos (UCI) debido a que aproximadamente el 12\% de los fallos anuales se presentan en esta área, en donde el $50 \%$ de estos fallos son el resultado de problemas de usabilidad. Este trabajo busca medir específicamente la interacción del personal médico con el equipo, empezando con una revisión bibliográfica, luego definiendo el equipo a trabajar. Finalmente, se propone desarrollar una propuesta de protocolo que permita evaluar la usabilidad del mismo y así sugerir algunos cambios que se pueden implementar.
\end{abstract}

Palabras clave — Monitoreo, sistema, UCI, usabilidad.

\section{Alarm Evaluation of an Intensive Care Unit Vital Signs Monitor System Based on Usability Parameters}

\footnotetext{
Abstract - This article presents a test model for the usability of a vital signs monitoring system, specifically in Intensive Care Units (ICUs). This is because approximately $12 \%$ of annual failures occur in this area and $50 \%$ of these are due to usability problems. The model specifically measures the interaction between medical personnel with the equipment, beginning with a bibliographical review, then defining the equipment with which to work to later suggest and develop a protocol proposal which will enable evaluating its usability and thus suggest some changes to be implemented.
}

Keywords - ICU, supervision, system, usability. 


\section{AVALIAÇÃO DOS ALARMES DE UM SISTEMA DE MONITORIZAÇÃO PARA UMA UNIDADE DE CUIDADOS INTENSIVOS E ESPECIAIS BASEANDO-SE EM CRITÉRIOS DE USABILIDADE}

Resumo - Neste artigo apresenta-se o aplicativo de um modelo de provas de usabilidade a um sistema de monitorização de signos vitais usados especificamente em unidades de cuidados intensivos (UCI) como aproximadamente o $12 \%$ das falhas anuais apresentam-se nesta área, e o $50 \%$ destas falhas são devido a problemas de usabilidade, medindo especificamente a interação pessoal médico com o equipamento, começando com uma revisão bibliográfica, logo definindo a equipe a trabalhar para posteriormente propor e desenvolver uma proposta de protocolo que permita avaliar a usabilidade do mesmo e assim sugerir algumas mudanças que podem-se implementar.

Palavras-chave - Monitorização, sistema, UCI, usabilidade

\section{INTRODUCCIÓN}

$\mathrm{L}^{\mathrm{a}}$ a usabilidad permite evaluar equipos y determinar qué tan amigables son con el usuario, factor que interviene en la tendencia a equivocarse durante la utilización del dispositivo. En este artículo se expondrá la metodología implementada para llegar a las pruebas de usabilidad, los resultados obtenidos con su respectivo análisis, para así llegar a las conclusiones del proyecto.

Al tener en cuenta criterios de usabilidad para evaluar un equipo, se está garantizando que este tenga una fácil interacción con el usuario, lo que lleva a una gran disminución en los errores que se puedan presentar con el uso cotidiano de un equipo [1]. Existe en algunos países reglamentación que exige la implementación de la usabilidad en dispositivos médicos, normas como la IEC 62366-1:2015 [2] establecen procesos para evaluar dispositivos con criterios de usabilidad enfocándose en la seguridad del paciente. Este proyecto aplicó evaluaciones de usabilidad las cuales fueron desarrolladas en base a la evaluación heurística establecida por Nielsen que tiene en cuenta al momento de evaluar componentes como: facilidad de aprendizaje, eficiencia, eficacia, cualidad de ser recordado y satisfacción del usuario [3].

La unidad de cuidados intensivos (UCI) es un servicio de alta complejidad cuyo objetivo es brindar un cuidado integral a aquellas personas en condiciones críticas de salud, que fueron internados allí [4].

Es por esto que la usabilidad orientada a este tipo de equipos juega un papel importante, ya que un mínimo error o una mínima falla ya sea debido al equipo o al personal médico puede poner en riesgo la vida del paciente [5]. En el artículo 'Error médico y eventos adversos' de Patricia Mena, se establece que cuando ocurren eventos adversos donde se compromete la seguridad del paciente, ya sea por error médico, falla del equipo o de usabilidad, el equipo médico es el responsable de prevenirlos, evitarlos e identificarlos oportunamente y limitar el daño al paciente [5]. Por lo anterior crece la necesidad de tener un control de alarmas y sobre el uso en general que se les hace a los equipos médicos dentro de un hospital y en especial dentro de una unidad de cuidados intensivos (UCI), mirando las fallas más repetitivas que se presentan dentro de este lugar y planteando posibles soluciones que van desde capacitaciones hasta el cambio del equipo manejado en dichas instalaciones.

\section{Metodología}

Para una evaluación en ingeniería de la usabilidad (UE), es necesario tener en cuenta varias consideraciones o aspectos que deben ser incluidos en la evaluación: Los usuarios, el entorno y las interfaces de usuario [6]. En el proyecto se implementó una metodología que incluyera las tres, los usuarios seleccionados para las pruebas debían ejercer en UCE o UCI que es el entorno a estudiar por los motivos mencionados anteriormente, además de estar en contacto con el equipo a evaluar, por esto se optó por escoger al técnico encargado de los equipos en UCI y al personal de enfermería de dicha área, este personal está capacitado para realizar tareas y manejar equipos que se encuentran en esta, y requieren de una preparación previa para poder trabajar en la UCI, y por último la interfaz de usuario evaluada será la del monitor de signos vitales.

El desarrollo del proyecto consistió en 4 etapas:

1. Antecedentes: Se comenzó por determinar qué normatividad se utiliza en Colombia en relación a la evaluación de equipos médicos, y cuál aplica específicamente para usabilidad. Se estableció el estado del arte en cuanto a artículos y estudios relacionados con el tema. Por último se determinó qué porcentaje de fallos en dispositivos estaban relacionados con usabilidad según reportes de tres años consecutivos de una base de datos de un hospital de Medellín a la cual se tuvo acceso durante el proyecto.

2. Definición de equipos a trabajar: Para esta etapa se decidió realizar la evaluación de un sistema de monitoreo completo, que incluye un monitor de signos vitales y la central de monitoreo que se encarga de supervisarlo. 
Usando criterios como tiempo para realizar una tarea, pasos, cualidad de recordación, efectividad del servicio, claridad en la interfaz y qué tan intuitiva es esta.

3. Propuesta protocolo y pruebas a realizar: Se realizaron diferentes pruebas relacionadas con la usabilidad desarrolladas en base a "Usability evaluation for a vital signs monitor prototype" [7].

- Caracterización alarmas del monitor de signos vitales usado dentro de la UCI.

- Comparación de la configuración de alarmas en una clínica de Medellín vs Configuración determinada (fábrica).

- Observación del manejo de alarmas en una UCI

- Prueba de manejo de alarmas a personal de enfermería para evaluar la usabilidad del equipo.

4. Análisis resultados: Con los resultados obtenidos se evaluó la usabilidad del equipo y la central, además se sugieren algunos cambios que podrían implementar en la UCI.

\section{Resultados}

Caracterización Monitor de signos vitales: Para el proyecto se dispuso de un monitor de signos vitales utilizado dentro de la UCI, el cual posee una plataforma de monitorización y un diseño totalmente modular que le permite medir y gestionar de forma simultanea un gran número de parámetros de monitorización, incluso modernas tecnologías como $\mathrm{PiCC} 02, \mathrm{ScvO} 2$ y $\mathrm{BISx} / \mathrm{x} 4$, así como herramientas clínicas como diagrama de araña y matriz espectral de densidad.

El protocolo HL7 y la pasarela de comunicación permiten al monitor dentro de la UCI interactuar a la perfección con el sistema de información del hospital o con un sistema de información clínica. La admisión de los paciente se realiza de forma sencilla, además, se pueden transferir al sistema de registro del paciente datos importantes de las constantes vitales. Este eficaz procedimiento, que se realiza sin papeleos, mejora la productividad y reduce el riesgo de errores de transcripción.

Con el módulo plug and play BeneLink, se pueden conectar al monitor hasta cuatro dispositivos, como ventiladores y máquinas de anestesia, lo que aumenta la cantidad de información que se suministra [8].

A este se le realizó una caracterización teniendo en cuenta parámetros de usabilidad. El primero a evaluar fue el sistema de colores, el cual si es adecuado facilita en gran medida la conexión del monitor, haciéndose más intuitiva para el usuario. Como se observa en la Fig. 1.
Tabla 1. Especificaciones monitor signos vitales

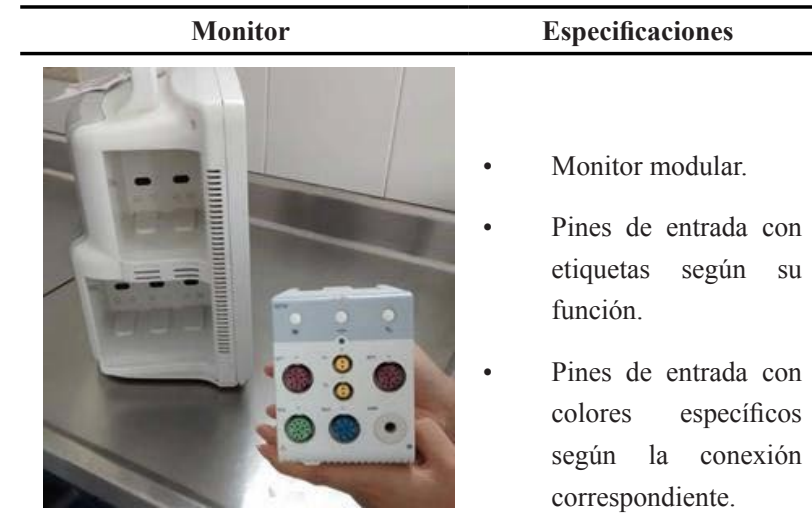

Fig. 1. Módulo para conexiones del monitor UCI

Las especificaciones de la Tabla 1 son beneficiosas para la usabilidad del equipo y por lo tanto contribuyen a la disminución de errores al momento de realizar la conexión de este.

Caracterización Central de Monitoreo: La central estaba ubicada en la Unidad de Cuidados Especiales (UCE) está ubicada estratégicamente, de manera que desde ella se tiene una visión de todos los cubículos. Esta cuenta con acceso a las variables otorgadas por el monitor de signos vitales. Esta información se visualiza constantemente en una pantalla tal como se observa en la Fig. 2.

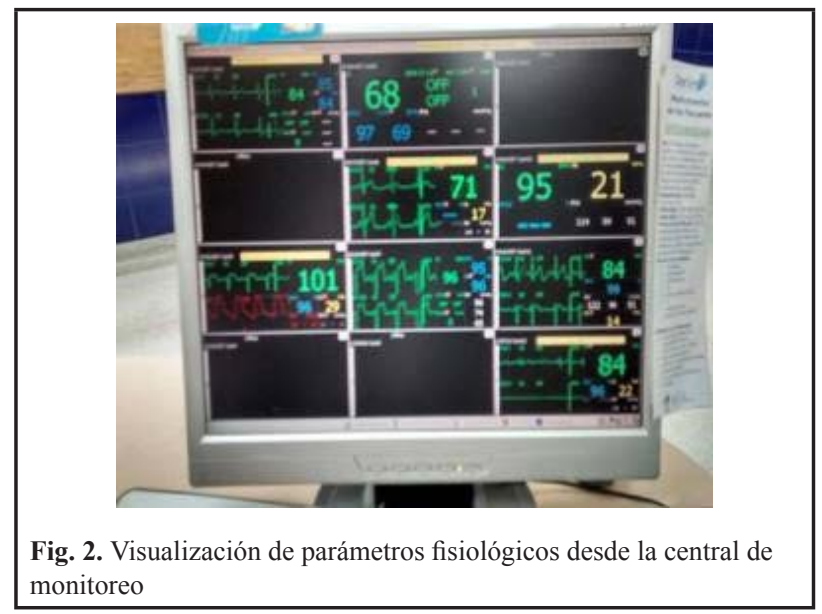

La central cuenta con sistema que relaciona una dirección propia de cada camilla y se la asigna al monitor, de esta forma el monitor envía su información especificando de qué dirección proviene. Esta dirección aparece en la parte superior de cada una de las divisiones correspondientes a cada monitor de signos vitales. La central de monitoreo caracterizada en este proyecto fue montada según el protocolo central monitoring system [9], donde se usó el protocolo para el montaje de red cableada. 
Caracterización alarmas monitor: En la Tabla 2 se puede observar una clasificación de las alarmas según su nivel de prioridad.

Tabla 2. Niveles alarmas monitor UCI

\begin{tabular}{ccc} 
Nivel & Alarmas fisiológicas & Alarmas Técnicas \\
\hline Alto & $\begin{array}{c}\text { Situación en la que la } \\
\text { vida del paciente se } \\
\text { encuentra en peligro }\end{array}$ & $\begin{array}{c}\text { Fallo grave del dispositivo en } \\
\text { el que el monitor puede no } \\
\text { detectar un estado crítico del } \\
\text { paciente }\end{array}$ \\
Medio & $\begin{array}{c}\text { Constantes del paciente } \\
\text { son anormales }\end{array}$ & $\begin{array}{c}\text { Fallo del dispositivo que no } \\
\text { pone en riesgo la vida del } \\
\text { paciente }\end{array}$ \\
Bajo & $\begin{array}{c}\text { Constantes del paciente } \\
\text { son anormales }\end{array}$ & $\begin{array}{c}\text { Mal funcionamiento del } \\
\text { dispositivo pero no pone en } \\
\text { riesgo la vida del paciente }\end{array}$ \\
\hline
\end{tabular}

En la Tabla 3 se pueden observar los diferentes indicadores de alarma con luz, que muestran las diferencias en luz, color y frecuencia de sonido dependiendo del nivel de prioridad de la alarma.

Tabla 3. Indicador de alarmas

\begin{tabular}{cccc}
\hline Nivel & Luz & Color & Sonido \\
\hline Alto & $\begin{array}{c}\text { La luz parpadea de } \\
\text { forma rápida }\end{array}$ & Rojo & Varias \\
Medio & $\begin{array}{c}\text { La luz parpadea de } \\
\text { forma lenta }\end{array}$ & Amarillo & 3 veces \\
Bajo & No parpadea & Amarillo & 1 vez \\
\hline
\end{tabular}

Comparación de la configuración de alarmas: Cuando se le asigna un monitor de signos vitales a un paciente, este es simplemente conectado adecuadamente con sus respectivos cables para las variables a medir, sin necesidad de variar la configuración para cada paciente. El único parámetro que se encontró diferente fue el volumen de las alarmas. Por fábrica, los monitores UCI, poseen la posibilidad de variar el volumen de las alarmas en un rango de 2 a 10 y vienen programados en el máximo volumen (en 10) de manera predeterminada siendo este el volumen recomendado por el proveedor. A pesar de esto se observó que los monitores se encontraban en el mínimo volumen de alarma (en 2) en la mayoría de los monitores que se encontraban en la UCI y en la UCE.

Observación alarmas: Para el seguimiento se tuvo en cuenta qué tipo de alarma generaba el monitor, su nivel de importancia y el tiempo que se demoraba el personal de enfermería para atender dicha alarma. Tras la realiza- ción de esta observación se determinó que es muy difícil determinar una referencia o llegar a alguna conclusión con este seguimiento. Dentro de las alarmas generadas por el monitor, varias se deben a que el paciente se mueve y existe una desconexión de algunos de los cables, para este caso el monitor emite una alarma de bajo o medio nivel de importancia. Esto puede llegar a suceder varias veces al día dependiendo del paciente, es por esto que en muchas ocasiones el personal de enfermería silencia estas alarmas mientras realizan otras actividades, lo cual representa un riesgo potencial para el paciente.

Prueba de usabilidad: Para esta prueba se conectó al monitor un simulador de ECG, con el cual se simularon valores por encima y por debajo de los límites de alarma, para poder activarlas y realizar pruebas con el personal del hospital.

Se realizó una prueba de usabilidad a 6 enfermeras del hospital dentro del área de UCI, adicionalmente se tuvo una referencia de evaluación, la cual correspondía a la persona encargada de la supervisión y mantenimiento de los equipos que se encuentran en la UCI.

Tabla 4. Resultados prueba usabilidad

\begin{tabular}{|c|c|c|c|}
\hline PERSONA & PRUEBA & TIEMPO & $\begin{array}{l}\text { NÚMERO } \\
\text { DE PASOS }\end{array}$ \\
\hline \multirow{3}{*}{ Referencia } & 2 & 0:07 & 1 \\
\hline & 3 & $0: 32$ & 3 \\
\hline & 4 & $0: 29$ & 3 \\
\hline \multirow{3}{*}{1} & 2 & $0: 45$ & 5 \\
\hline & 3 & \multicolumn{2}{|c|}{ No sabía realizar la acción } \\
\hline & 4 & $2: 45$ & 21 \\
\hline \multirow{3}{*}{2} & 2 & $0: 34$ & 7 \\
\hline & 3 & $1: 50$ & 12 \\
\hline & 4 & $0: 22$ & 2 \\
\hline \multirow{3}{*}{3} & 2 & $0: 39$ & 1 \\
\hline & 3 & $0: 30$ & 3 \\
\hline & 4 & $0: 22$ & 3 \\
\hline \multirow{3}{*}{4} & 2 & $0: 14$ & 1 \\
\hline & 3 & $0: 39$ & 4 \\
\hline & 4 & $1: 17$ & 3 \\
\hline \multirow{3}{*}{5} & 2 & $0: 17$ & 1 \\
\hline & 3 & $0: 52$ & 8 \\
\hline & 4 & $0: 14$ & 3 \\
\hline \multirow{3}{*}{6} & 2 & $0: 25$ & 1 \\
\hline & 3 & $0: 40$ & 8 \\
\hline & 4 & $0: 16$ & 3 \\
\hline
\end{tabular}


La prueba constaba de cuatro ítems:

1. Identificar el nivel de la alarma que estaba activada.

2. Silenciar la alarma.

3. Cambiar los valores de los límites de dicha alarma.

4. Cambiar el volumen de la alarma.

Los resultados obtenidos de la prueba para los puntos 2, 3, y 4 se ven condensados en la Tabla 4 .

En la Tabla 5 se observan los resultados para el ítem 1, donde se evalúa la identificación de la alarma.

Tabla 5. Resultados prueba de usabilidad (pregunta 1)

\begin{tabular}{cc}
\hline & ITEM 1 \\
\hline PERSONA & NIVEL DE ALARMA \\
Referencia & Medio \\
1 & Bajo \\
2 & Bajo \\
3 & Alto \\
4 & Medio \\
5 & Medio-Bajo \\
6 & Alto \\
\hline
\end{tabular}

\section{DisCUSIÓN}

Caracterización Central de Monitoreo: Dentro de los inconvenientes encontrados en cuanto a la usabilidad de la central de monitoreo es que según el protocolo que se usa en la clínica para el montaje de esta [1], la central usa el protocolo para una central de monitoreo cableada. Se encontró que durante las rondas de observación que se realizaron en la UCE, que se aflojaba la conexión de algunos cables en los monitores, o en los pines de entrada en el monitor que está ubicado en la estación de enfermería, por lo que no se podía visualizar en pantalla la información correspondiente a todos los pacientes. Cuando esto sucede, el enfermero jefe se encarga de informar al técnico para verificar las conexiones, ajustar los cables y así solucionar el problema. Lo anterior puede deberse a un desgaste de los cables por el tiempo, lo que va disminuyendo la usabilidad de la central puesto que los usuarios que la utilizan tienen dificultades y se ven obligados a llamar a los técnicos para encontrar soluciones. Es por esto que para incrementar la usabilidad de esta central, se propone modificar el protocolo actual por uno que se lleve a cabo de manera inalámbrica, para eliminar posibles alarmas que pudieron ser desatendidas porque en la central de monitoreo no se visualizaba un paciente específico.
Caracterización alarmas monitor: El caso omiso de una atención de una alarma puede poner en riesgo la vida del paciente, puesto que estas alarmas son generadas cuando hay un parámetro fisiológico fuera de los rangos recomendados. Anteriormente se habló sobre los niveles y causas por las cuales estas alarmas se activan, en el caso de una alarma de nivel alto que se active por una falla técnica del monitor como lo es batería baja, si el personal médico no está capacitado para comprender y solucionar el problema simplemente porque el paciente no tiene alterados sus signos vitales, puede ocasionar la detención del monitor y por ende la visualización del estado del paciente lo que puede conllevar a un sin fin de eventos los cuales pudieron ser evitados por una buena detección y solución de la alarma.

Los indicadores visuales o acústicos son una alternativa útil a la hora de clasificar la alarma para una adecuada atención de la misma, ya que identificando el color de la alarma se sabrá qué tan crítica es la situación para la solución de esta. Al caracterizar las alarmas con las cuales viene el equipo y comparándolas con las configuraciones con las que trabajan en dicha unidad de servicios se identificó que el ajuste del volumen de alarma a un nivel bajo puede resultar en una situación peligrosa para el paciente ya que un cambio repentino en los signos vitales de un paciente puede no ser escuchado por el personal para tomar medidas, como se mencionó anteriormente el equipo viene por fábrica con el volumen máximo (10) y se observó que los equipos evaluados, trabajan al volumen mínimo(2), siendo esta una advertencia hecha por el fabricante.

Pruebas de usabilidad: Al realizar la prueba de usabilidad se observó que hay variación de los datos respecto a la referencia:

Respecto a la prueba 1, la cual correspondía en decir cuál era el nivel de la alarma que arrojaba el monitor la respuesta era cualitativa y no se tomaban ni tiempo ni pasos utilizados para realizar dicha tarea, se encontró que solo dos de las seis personas respondieron correctamente a dicha pregunta y esto puede deberse a que el nivel de alarma bajo y medio se activan del mismo color y lo único en lo que difieren es en el sonido y las veces que titilan, respecto a las personas que contestaron nivel alto no entendieron bien la pregunta y se confundieron con el límite de alarma de los signos vitales mas no del nivel de la misma, sin embargo el saber o no el nivel de la alarma juega un papel importante a la hora de tomar la decisión de acudir al médico de guardia debido que estos niveles nos dicen que tan grave es la situación y si el paciente necesita atención de inmediato.

Respecto a la prueba 2, que correspondía en silenciar la alarma emitida anteriormente la referencia de respuesta es de 1 paso y tomo 7 segundos. Según la Tabla 4 se 
evidencia que la mayoría de las personas que tomaron la prueba lo hicieron en los pasos adecuados respecto a la referencia, este comportamiento se debe a que en el monitor tiene en su interfaz un botón con el cual esta acción se realiza inmediatamente y por esto no toma ni mucho tiempo ni muchos pasos realizarla, y en menos de 1 minuto es posible realizarla.

Respecto a la prueba 3, la cual correspondía a cambiar los parámetros de límites de alarma de ECG, se tiene como referencia un tiempo de 32 segundos y 3 pasos para realizar dicha tarea. Se encontró que casi todas las personas se tomaron más del tiempo y pasos respecto a la referencia, debido a que al equipo en ningún momento se le modifican estas variables sino que trabajan con las dadas de fábrica y por esto no están familiarizadas con esta función, sin embargo la importancia de conocer y manipular los límites de alarma son muy importantes a la hora de interpretar la enfermedad de un paciente, es decir, algunos pacientes con patologías relacionadas a cardiopatías se les debe dar una mayor prioridad a los cambios de sus signos vitales y requieren de una modificación de esta función para vigilar mejor sus variables y poder responder a tiempo a algún evento.

Respecto a la prueba 4, la cual correspondía a cambiar el nivel de volumen del monitor, se tomó como referencia un tiempo empleado de 24 segundos y 5 pasos. Se observa que esta es la tarea que realizan con mayor facilidad, como se mencionó anteriormente este parámetro es modificado respecto al de fábrica y por esto están más familiarizados con esta modificación respecto a otras. Sin embargo se recalca que este parámetro no debería ser modificado ya que podría desencadenar una situación peligrosa para el paciente.

Con base a los resultados anteriores se recomienda realizar una capacitación al personal sobre el monitor de signos vitales UCI, resaltando la importancia de conocer funciones tales como la modificación del límite de alarmas ya que aunque esto no es muy común que se realice se debe conocer muy bien el equipo y todas las funciones que este posee, además se debe enfatizar en la importancia de la identificación de alarmas, puesto que al haber diferentes tipos no solo fisiológicas sino también técnicas se debe conocer y saber solucionarlas para así evitar un evento adverso. Se encontró que de acuerdo a los colores y sonidos propios de las alarmas del monitor UCI, existe mucha similitud en las alarmas de nivel bajo y medio, y el personal de enfermería al que se evaluó mostró dificultad para diferenciarlas, una posible solución podría ser que las alarmas varíen también de color en estos tres niveles, para que la distinción entre ellas sea más clara. Debido al gran número de alarmas que se perciben diariamente en una unidad de cuidados intensivos se evidenció que muchos monitores son silenciados o que configuran su volumen en lo mínimo, generado así situaciones potencialmente riesgosas para el paciente. Además de las muchas alarmas de niveles bajos o medios que se presentan diariamente, el personal de enfermería tiene diferentes ocupaciones en su jornada laboral; La digitalización de todo lo que ocurre con un paciente requiere de tiempo, es por esto que cuando el personal detecta la activación de una alarma de bajo nivel, y decide silenciarla mientras termina alguna otra de sus labores. Entre estos intervalos de tiempo se prestan espacios de desatención del paciente que pueden ser críticos.

Un cambio en la implementación de las alarmas del monitor y también en la capacitación del personal de enfermería podría disminuir los riesgos que esto representa.

\section{Conclusiones}

- La unidad de cuidados intensivos (UCI) es un sistema que requiere la atención y manejo adecuado ya que allí se encuentran los pacientes más críticos, por esto la importancia de la detección prudente de las alarmas arrojadas por los monitores de signos vitales, puesto que la más mínima alteración de algunos de estos parámetros puede ocasionar la muerte del paciente.

- Es de vital importancia que en las unidades hospitalarias se realicen frecuentemente capacitaciones donde el personal interactúe con el equipo y realice funciones determinadas, así el personal estará preparado para cualquier daño, alarma, desconfiguración, entre otras, que pongan en riesgo la vida del paciente y puedan ser solucionadas de inmediato.

- Las alarmas representan uno de los puntos críticos en el tema de seguridad del paciente es por esto que se vuelve una necesidad evaluar la usabilidad de los equipos que emiten alarmas, y la evaluación de estas con el fin de proponer estrategias que ayuden a disminuir los errores presentados por mal manejo de alarmas.

- Herramientas como las pruebas de usabilidad pueden ayudar a establecer una mejor panorámica acerca de qué medidas tomar respecto a la disminución de los errores en un servicio, ya sea un cambio de equipo o mayor capacitación para el personal, si se tiene información acerca de la usabilidad de un equipo se pueden proponer mejores enfoques para comprender y tratar un problema con este.

\section{Agradecimientos}

Este trabajo fue soportado parcialmente por el Sistema General de Regalías, de la República de Colombia, a través del proyecto "Fortalecimiento de la plataforma tecnológica para la formación especializada en el área de 
la salud y el desarrollo de tecnología biomédica", código RutaN-139C

\section{REFERENCIAS}

[1]. H. Luini, O. Castrillón and G. Olivar, "An Automated Methodology for Usability Evaluation of Interfaces of Industrial Supervision”, Información Tecnológica, vol. 4, pp. 95-104, 2013.

[2]. International Organization for Standardization (2016, 5 April). IEC 62366-1:2015 [Online]. Available: http://www.iso.org/iso/ catalogue_detail.htm?csnumber $=63179$

[3]. J. Jeng, "Usability assessment of academic digital libraries: effectiveness, efficiency, satisfaction, and learnability", LIBRI, vol. 55, pp. 96-121, 2005.

[4]. V. A. Baena and S. M León, "El temor y la experiencia del paciente adulto críticamente enfermo hospitalizado en la unidad de cuidado intensivo", Tesis Especialización en cuidado de enfermería al adulto en estado crítico en salud. Fac. Enfermería, Antioquia Univ., Medellín 2007.

[5]. P. Mena, "Error médico y eventos adversos", Revista Chilena de Pediatría, vol. 79, pp. 319-326, Mayo 2008.

[6]. J. C. Olivera, (2016, Septiembre), Análisis de interfaz de usuario en bombas de infusión: Un enfoque sobre usabilidad, ReseaechGate [Online], Available: https://www.researchgate. net/profile/Juan_Olivera2/publicat ion/252930878_Anlisis_de interfaz_de_usuario_en_bombas_de_infusin_Un_enfoque_sobre usabilidad/links/00b4951f5339275911000000.pdf

[7]. L. Arenas, P. Bedoya, L. Correa, J. Barreneche and A. Hernández., "Usability evaluation for a vital signs monitor prototype", presentado en el Congreso CLAIB 2016, Burcaramanga, 2016.

[8]. Mindray. (2017, Enero). BeneView T5/T6/T8/T9 [Online]. Available: http://www.mindray.com/es/product/BeneView_T5.or. T6.or.T8.or.T9.html

[9]. Mindray. (2016, Noviembre). HYPERVISOR VI Central Monitoring System [Online]. Available: http://www.infiniti. se/upload/Mindray/Manualer/CMS/MIN SM_EN_CMS HYPERVISOR\%20VI_Service\%20Manual_V10.0_English.pdf 